\title{
corso pre-congressuale
}

\section{SESSIONE CONGIUNTA AMCLI/SIMPIOS}

\section{Prevenzione e sorveglianza delle infezioni nelle organizzazioni sanitarie: quale ruolo per il microbiologo?}

\author{
Martedi I I Ottobre 2005, ore I4.00 - 17.00, Sala E
}

\section{INFEZIONI NELLE STRUTTURE SANITARIE: STATO DELL'ARTE}

\section{Moro M.L. \\ Agenzia Sanitaria Regionale, Bologna}

Vi sono molti motivi per cui le infezioni associate a pratiche assistenziali devono essere considerate una priorità di sanità pubblica: a) il rischio di contrarre una infezione nel corso dell'assistenza ospedaliera è in continuo aumento, parallelamente all'incremento della popolazione ricoverata altamente suscettibile alle infezioni (trapiantati, neonati prematuri, ecc.); b) i luoghi a rischio si sono moltiplicati come conseguenza dei cambiamenti nei modelli assistenziali (strutture residenziali, lungodegenza, assistenza domiciliare, daysurgery, ecc.); c) l'assistenza "in rete" aumenta il rischio di trasmissione dall'ospedale in comunità e da questa all'ospedale di infezioni sostenute da microrganismi antibiotico-resistenti; d) la "globalizzazione" aumenta il rischio di diffusione a livello mondiale di infezioni emergenti in particolari aree del mondo, che possono trasmettersi all'interno dell'ospedale, come si è verificato per la SARS nel 2003.

Parallelamente, vi sono spinte all'interno dei sistemi sanitari, per affrontare con impegno il problema della sicurezza dei servizi sanitari, la gestione in modo appropriato ed efficace delle risorse disponibili, la trasparenza nei confronti degli utenti. Tutto ciò ha una ricaduta positiva anche per i programmi di controllo delle infezioni nelle strutture sanitarie.

L'Organizzazione Mondiale della Sanità, ad esempio, ha lanciato nel 2004 la World Alliance for Patient Safety il cui motto è "Clean care is safer care" e che si pone come primo obiettivo la promozione di interventi mirati a migliorare l'appropriatezza della igiene delle mani in tutto il mondo.

In Italia, diverse regioni hanno attivato programmi di controllo organici delle infezioni nelle strutture sanita- rie, che hanno affrontato sia il tema della sorveglianza che quello del controllo. In particolare, alcuni regioni hanno recentemente realizzato studi di prevalenza per quantificare il fenomeno delle infezioni in ospedale (ad es. Lombardia, Piemonte, Veneto, Toscana) o in strutture residenziali per anziani (ad es. Emilia-Romagna). Sono stati attivati sistemi di sorveglianza a partire da fonti informative correnti, quali i laboratori di microbiologia (ad es. Veneto ed Emilia-Romagna), in alcuni casi analizzati in modo integrato con le Schede di Dimissione Ospedaliera (Veneto). Sono stati avviati sistemi regionali di sorveglianza di specifiche infezioni, quali quelle del sito chirurgico (Friuli Venezia Giulia) o delle polmoniti associate a ventilazione (FVG e Piemonte). Sono stati attuati audit delle misure di controllo in reparti a rischio, quali i reparti chirurgici ed i blocchi operatori (ad es. Piemonte ed Emilia-Romagna) o le unità di terapia intensiva (Emilia-Romagna).

Per garantire il coinvolgimento diretto di tutte le Aziende Sanitarie si è fatto leva sulla valutazione di risultato dei Direttori Generali (FVG e Piemonte) o sui requisiti per l'autorizzazione e l'accreditamento delle strutture (Emilia-Romagna).

Rispetto a vent'anni fa sono stati fatti molti passi in avanti, ma è necessario un maggior coordinamento delle iniziative e la loro diffusione a livello nazionale. 